\title{
SCATTERING OF FERMIONS BY A MAGNETIC DIPOLE FIELD
}

\author{
Sergiu Hategan ${ }^{\mathrm{a}, *}$ and Cosmin Crucean ${ }^{\mathrm{a}, \dagger}$ \\ ${ }^{a}$ Faculty of Physics, West University of Timișoara \\ 300223 V. Pârvan Ave 4, Timișoara, Romania \\ *sergiu.hategan98@e-uvt.ro \\ $\dagger$ crucean@physics.uvt.ro (corresponding author)
}

\begin{tabular}{|l|l|}
\hline Article info & \multicolumn{1}{c|}{ Abstract } \\
\hline Received: 17.10 .2021 & $\begin{array}{l}\text { In this paper we study the problem of fermions scattering by the } \\
\text { field of a magnetic dipole in Minkowski space-time. The amplitude } \\
\text { and } 10.11 .2021\end{array}$ \\
$\begin{array}{l}\text { Keywords: Magnetic dipole, } \\
\text { First order amplitudes, Differen- } \\
\text { tial cross section. }\end{array}$ & $\begin{array}{l}\text { obtained using the exact solution of the Dirac equation written in the } \\
\text { helicity basis. We found that the most probable transitions are those } \\
\text { that scatter the fermions perpendicular to the direction of the magnetic } \\
\text { field and we consider only the transverse momenta in our analysis. } \\
\text { The differential cross section behavior in terms of scattering angle } \\
\text { and energy is graphically analysed and we perform a comparative } \\
\text { study with the Coulomb scattering. }\end{array}$ \\
\hline
\end{tabular}

\section{Introduction}

The scattering of massless Dirac fermions by spatially localized static magnetic fields was studied in [1], where the effects of orbital magnetic fields, Zeeman and exchange fields in topological insulators, and the pseudomagnetic fields caused by defects in mono- layer graphene were considered. In [1] the scattering theory was constructed and the scattering amplitude and the total cross sections were obtained in terms of phase shifts for radially symmetric fields. Another result is related to the scattering calculation that probes the anapole moment with a spinless charged particle using the quantum field theory [2]. In [2] it was proven that in the non-relativistic limit the scattering cross section agrees with the cross section obtained in quantum mechanics for a spinless current scattered by an infinitesimally thin toroidal solenoid.

In the present paper we want to analyse the result obtained directly from the first order scattering amplitude of Minkowski QED in what concerns the fermions scattering on the dipole magnetic field. The definition of potential vector in terms of magnetic moment will be used for computing the first order transition amplitude of fermions scattering. An example of transition amplitude with the dipole magnetic field can be found in [3] where the phenomenon of particle production was investigated in a non-stationary metric. The dipole magnetic field was used 
in limited computations due to the dependence of $r^{-3}$ which lead to complications in effective calculations. In this paper we will use the plane wave solutions of the Dirac equation written in the helicity basis on Minkowski space-time. We also must specify that the analysis is restricted to the transverse momenta and the analysis for the general case is not performed in our paper. Our results prove that the scattering at small angles is dominant when we take into account that the fermions will be scattered perpendicular with the direction of magnetic field.

In the second section we present the main steps for computing the scattering amplitude. The third section is dedicated to the computation of differential cross section and in the four section we perform a graphical analysis of the cross section in terms of scattering angle and energy. In our computations we use the Minkowski metric $\eta^{\mu \nu}=d t^{2}-d \vec{x}^{2}$ and the natural units $\hbar=1, c=1$.

\section{Scattering in a magnetic dipole field}

The Coulomb scattering is the most studied example of a process in the first order of perturbation theory in Minkowski QED. An interesting result could be also obtained if we study the fermions scattering on vector potentials that generate magnetic fields. One such example is the fermion scattering on dipole magnetic field that could be studied as a first order perturbative process in Minkowski QED. The first order transition amplitude in Minkowski QED, written in terms on the fundamental spinor solutions $U_{p \sigma}(x)$ of the Dirac equation is [4]:

$$
A_{i \rightarrow f}=-i e \int d^{4} x \bar{U}_{p^{\prime} \sigma^{\prime}}(x) \gamma^{\mu} \cdot A_{\mu}(x) U_{p \sigma}(x)
$$

where $A_{i \rightarrow f}$ stands for the transition amplitude from the initial state to the final state and $\gamma^{\mu}$ are the Dirac matrices. For a magnetic dipole, the potential $A_{\mu}$ only has spatial components, described by the vector potential [5]:

$$
\vec{A}(x)=\frac{\vec{M} \times \vec{x}}{|\vec{x}|^{3}}
$$

Then the scattering amplitude with the above potential is:

$$
A_{i \rightarrow f}=-i e \int d^{4} x \bar{U}_{p^{\prime} \sigma^{\prime}}(x) \vec{\gamma} \cdot \vec{A}(x) U_{p \sigma}(x)
$$

In this calculation the solutions of the Dirac equation will be expressed in the helicity basis [4]:

$$
U_{p \sigma}(x)=\sqrt{\frac{m}{E(2 \pi)^{3}}} \frac{1}{\sqrt{2 m(E+m)}}\left(\begin{array}{c}
(E+m) \xi_{\sigma} \\
2 \sigma p \xi_{\sigma}
\end{array}\right) e^{-i p x},
$$

where the unit normalized helicity spinors are :

$$
\xi_{\frac{1}{2}}(\vec{p})=\sqrt{\frac{p_{3}+p}{2 p}}\left(\begin{array}{c}
1 \\
\frac{p_{1}+i p_{2}}{p_{3}+p}
\end{array}\right), \quad \xi_{-\frac{1}{2}}(\vec{p})=\sqrt{\frac{p_{3}+p}{2 p}}\left(\begin{array}{c}
\frac{-p_{1}+i p_{2}}{p_{3}+p} \\
1
\end{array}\right) .
$$


The amplitude of this process is:

$$
\begin{aligned}
A_{i \rightarrow f}=-i e \int d^{4} x & \frac{m}{(2 \pi)^{3} 2 m \sqrt{E E^{\prime}\left(E^{\prime}+m\right)(E+m)}} e^{-i\left(\overrightarrow{p^{\prime}}-\vec{p}\right) \cdot \vec{x}} e^{i\left(E^{\prime}-E\right) t} . \\
\left((E+m) \xi_{\sigma^{\prime}}^{\dagger}\right. & \left.2 \sigma^{\prime} p^{\prime} \xi_{\sigma^{\prime}}^{\dagger}\right)\left(\begin{array}{cc}
1 & 0 \\
0 & -1
\end{array}\right)\left(\begin{array}{cc}
0 & \vec{\sigma} \\
-\vec{\sigma} & 0
\end{array}\right) \frac{\vec{M} \times \vec{x}}{|\vec{x}|^{3}}\left(\begin{array}{c}
(E+m) \xi_{\sigma} \\
2 \sigma p \xi_{\sigma}
\end{array}\right)
\end{aligned}
$$

where $E^{\prime}, p^{\prime}$ are the final values of the momentum and energy, after the scattering process. The temporal integral will give the result $2 \pi \delta\left(E^{\prime}-E\right)$, and the space integral can be considered as being the Fourier transform of the function $\frac{\vec{x}}{|\vec{x}|^{3}}$, and this function is in turn $\frac{\vec{x}}{|\vec{x}|^{3}}=\nabla\left(\frac{1}{\vec{x}}\right)$. Thus from the properties of the Fourier transform it results that the integral is equal to:

$$
\int d^{3} x \frac{\vec{x}}{|\vec{x}|^{3}} e^{-i\left(\overrightarrow{p^{\prime}}-\vec{p}\right) \cdot \vec{x}}=-i\left(\overrightarrow{p^{\prime}}-\vec{p}\right) \int d^{3} x \frac{1}{|\vec{x}|} e^{-i\left(\overrightarrow{p^{\prime}}-\vec{p}\right) \cdot \vec{x}}
$$

The second integral has the known result $\frac{4 \pi}{\left|\vec{p}^{\prime}-\vec{p}\right|^{2}}$, therefore the space integral has the final result:

$$
\int d^{3} x \frac{\vec{x}}{|\vec{x}|^{3}} e^{-i\left(\overrightarrow{p^{\prime}}-\vec{p}\right) \cdot \vec{x}}=-\frac{4 i \pi\left(\vec{p}^{\prime}-\vec{p}\right)}{\left|\overrightarrow{p^{\prime}}-\vec{p}\right|^{2}}
$$

With the above results for the temporal and spatial integrals the amplitude takes the following form:

$$
\begin{aligned}
A_{i \rightarrow f}=\frac{-e 2 \pi \delta\left(E^{\prime}-E\right)(4 \pi)}{(2 \pi)^{3}\left|\overrightarrow{p^{\prime}}-\vec{p}\right|^{2} \sqrt{E^{\prime} E\left(E^{\prime}+m\right)(E+m)}}\left[2 \sigma^{\prime} p^{\prime}(E+m)+2 \sigma p\left(E^{\prime}+m\right)\right] \\
\times \xi_{\sigma^{\prime}}^{\dagger} \vec{\sigma} \cdot\left(\vec{M} \times\left(\overrightarrow{p^{\prime}}-\vec{p}\right)\right) \xi_{\sigma}
\end{aligned}
$$

Considering the values $\sigma^{\prime}, \sigma$ to be $\pm \frac{1}{2}$, we can rewrite $2 \sigma=\operatorname{sgn}(\sigma)$, using the signum function:

$$
\begin{aligned}
& A_{i \rightarrow f}=\frac{-e 2 \pi \delta\left(E^{\prime}-E\right)(4 \pi)}{(2 \pi)^{3}\left|\overrightarrow{p^{\prime}}-\vec{p}\right|^{2} \sqrt{E^{\prime} E\left(E^{\prime}+m\right)(E+m)}}\left[\operatorname{sgn}\left(\sigma^{\prime}\right) p^{\prime}(E+m)+\right.\left.\operatorname{sgn}(\sigma) p\left(E^{\prime}+m\right)\right] \\
& \times \xi_{\sigma^{\prime}}^{\dagger} \vec{\sigma} \cdot\left(\vec{M} \times\left(\overrightarrow{p^{\prime}}-\vec{p}\right)\right) \xi_{\sigma} .
\end{aligned}
$$

The conservation of energy in this process is assured by the delta Dirac term $\delta\left(E^{\prime}-E\right)$. Let us evaluate the term dependent of helicity spinors. First the magnetic dipole moment will be considered to be oriented on the third axis, $\vec{M}=M \vec{e}_{3}$ and it is possible to work in polar coordinates with the components of the momentum on the 1 and 2 axes, when calculating the cross product from the integral:

$$
\vec{M} \times\left(\vec{p}^{\prime}-\vec{p}\right)=M\left(\sigma_{2}\left(p_{1}^{\prime}-p_{1}\right)-\sigma_{1}\left(p_{2}^{\prime}-p_{2}\right)\right)
$$


Changing from cartesian coordinates to spherical coordinates is given by the relations:

$$
\begin{array}{ll}
p_{1}{ }^{\prime}=p^{\prime} \sin \alpha \cos \beta & p_{1}=p \sin \gamma \cos \lambda \\
p_{2}{ }^{\prime}=p^{\prime} \sin \alpha \sin \beta & p_{2}=p \sin \gamma \sin \lambda \\
p_{3}{ }^{\prime}=p^{\prime} \cos \alpha & p_{3}=p \cos \gamma
\end{array}
$$

In polar coordinates $\alpha=\gamma=\frac{\pi}{2}$, and it follows:

$$
\begin{array}{ll}
p_{1}{ }^{\prime}=p^{\prime} \cos \beta & p_{1}=p \cos \lambda \\
p_{2}{ }^{\prime}=p^{\prime} \sin \beta & p_{2}=p \sin \lambda \\
p_{3}{ }^{\prime}=0 & p_{3}=0
\end{array}
$$

The product $\xi_{\sigma^{\prime}}^{\dagger} \vec{\sigma} \cdot\left(\vec{M} \times\left(\vec{p}^{\prime}-\vec{p}\right)\right) \xi_{\sigma}$ can now be calculated, for equal values of $\sigma$, the case when the helicity is conserved after the scattering, using the following values for $\xi_{\sigma}$ :

$$
\xi_{1 / 2}(\vec{p})=\frac{1}{\sqrt{2}}\left(\begin{array}{c}
1 \\
e^{i \beta}
\end{array}\right), \quad \xi_{-1 / 2}(\vec{p})=\frac{1}{\sqrt{2}}\left(\begin{array}{c}
-e^{-i \beta} \\
1
\end{array}\right)
$$

where $\beta$ is the polar angle corresponding to momentum $p^{\prime}$, and $\lambda$ the angle corresponding to the momentum $p$. When the values of $\sigma, \sigma^{\prime}$ are equal, namely $\sigma=\sigma^{\prime}=1 / 2$, we obtain:

$$
\xi_{1 / 2}^{\dagger} \sigma_{2}\left(p_{1}^{\prime}-p_{1}\right) \xi_{1 / 2}-\xi_{1 / 2}^{\dagger} \sigma_{1}\left(p_{2}^{\prime}-p_{2}\right) \xi_{1 / 2}=\frac{i}{2}\left(p^{\prime}+p\right)\left(1-e^{-i(\beta-\lambda)}\right)
$$

Taking the squared modulus of the product gives:

$$
\frac{1}{4}\left(p^{\prime}+p\right)^{2}\left(1+1-e^{i(\beta-\lambda)}-e^{-i(\beta-\lambda)}\right)=\frac{1}{2}\left(p^{\prime}+p\right)^{2}(1-\cos (\beta-\lambda))
$$

The same derivation is repeated for opposite values of $\sigma$, when helicity is not conserved; $\sigma=1 / 2, \sigma^{\prime}=-1 / 2$ :

$$
\xi_{-1 / 2}^{\dagger} \sigma_{2}\left(p_{1}^{\prime}-p_{1}\right) \xi_{-1 / 2}-\xi_{1 / 2}^{\dagger} \sigma_{1}\left(p_{2}^{\prime}-p_{2}\right) \xi_{1 / 2}=\frac{i}{2}\left(p^{\prime}-p\right)\left(e^{i \beta}+e^{i \lambda}\right)
$$

Taking the squared modulus gives this result:

$$
\frac{1}{4}\left(p^{\prime}-p\right)^{2}\left(2+e^{i(\beta-\lambda)}+e^{-i(\beta-\lambda)}\right)=\frac{1}{2}\left(p^{\prime}-p\right)^{2}(1+\cos (\beta-\lambda))
$$

This term is vanishing for $p^{\prime}=p$, because momentum and energy are conserved as shown by the delta Dirac function in the amplitude. This term will not be added when summing by helicities, being equal to zero. Taking the spin averaged matrix element squared and considering the nonvanishing contributions, $\sigma=\sigma^{\prime}=1 / 2$ and $\sigma=\sigma^{\prime}=-1 / 2$, and disregarding the ones 
for opposite helicities, we obtain for $E=E^{\prime}$ or $p=p^{\prime}$ :

$$
\begin{aligned}
\left.\frac{1}{2} \sum_{\sigma \sigma^{\prime}}\left|A_{i \rightarrow f}\right|^{2}\right|_{\sigma} & =\sigma^{\prime} \\
& =\frac{M^{2} e^{2}\left(2 \pi \delta\left(E^{\prime}-E\right)\right)^{2} 16 \pi^{2}\left(2 m+E+E^{\prime}\right)^{2}}{(2 \pi)^{6} E^{\prime} E\left(E^{\prime}+m\right)(E+m)\left|\overrightarrow{p^{\prime}}-\vec{p}\right|^{4}}\left(p^{\prime}+p\right)^{2}(1-\cos (\beta-\lambda))
\end{aligned}
$$

\section{Differential cross section}

In order to obtain a cross section the transition rate must be found, by integrating the final momentum, and dividing this quantity by the incident flux, calculated using the spatial component of the Dirac current.

The transition rate is defined by taking the derivation of probability with respect to time:

$$
\frac{d P_{i \rightarrow f}}{d t}=\frac{4 M^{2} e^{2} \delta\left(E^{\prime}-E\right)\left(2 m+E+E^{\prime}\right)^{2}}{(2 \pi)^{3} E^{\prime} E\left(E^{\prime}+m\right)(E+m)\left|\vec{p}^{\prime}-\vec{p}\right|^{4}}\left(p^{\prime}+p\right)^{2}(1-\cos (\beta-\lambda))
$$

The differential cross section is obtained dividing by spherical measure and integrating after the modulus of the final momenta:

$$
\begin{array}{r}
\frac{d \sigma}{d \Omega}=\int \frac{R_{i \rightarrow f}}{F_{i}} p^{\prime 2} d p^{\prime}=\int \frac{(2 \pi)^{3} E}{p} \frac{4 M^{2} e^{2} \delta\left(E^{\prime}-E\right)\left(2 m+E+E^{\prime}\right)^{2}}{(2 \pi)^{3} E^{\prime} E\left(E^{\prime}+m\right)(E+m)\left|\vec{p}^{\prime}-\vec{p}\right|^{4}} \\
\times\left(p^{\prime}+p\right)^{2}[1-\cos (\beta-\lambda)]\left(p^{\prime}\right)^{2} d p^{\prime}
\end{array}
$$

Using the variable change $p^{\prime} d p^{\prime}=E^{\prime} d E^{\prime}$, it is obtained:

$$
\frac{d \sigma}{d \Omega}=\frac{4 M^{2} e^{2}}{p} \int d E^{\prime} E^{\prime} p^{\prime} \frac{\delta\left(E^{\prime}-E\right)\left(2 m+E+E^{\prime}\right)^{2}}{E^{\prime}\left(E^{\prime}+m\right)(E+m)\left|\vec{p}^{\prime}-\vec{p}\right|^{4}}\left(p^{\prime}+p\right)^{2}(1-\cos (\beta-\lambda))
$$

Integrating the delta Dirac function $\left(E^{\prime}=E, p^{\prime}=p\right)$ gives:

$$
\frac{d \sigma}{d \Omega}=\frac{4 M^{2} e^{2} p(2 m+2 E)^{2}}{p(E+m)^{2}\left|\vec{p}^{\prime}-\vec{p}\right|^{4}} \cdot 4 p^{2}(1-\cos (\beta-\lambda))
$$

After simplifying and using the relation $\left|\vec{p}^{\prime}-\vec{p}\right|^{4}=16 p^{4} \sin ^{4} \frac{\theta}{2}$, with scattering angle $\theta=\beta-\lambda$ gives the formula:

$$
\frac{d \sigma}{d \Omega}=\frac{8 M^{2} e^{2}}{p^{2} \sin ^{2} \frac{\theta}{2}}
$$

This can be expressed as a function of $\beta$ in this way:

$$
\frac{d \sigma}{d \Omega}=\frac{8 M^{2} e^{2}}{\beta^{2} E^{2} \sin ^{2} \frac{\theta}{2}}
$$




\section{Graphic results}

In this section we present a comparison of the differential cross section for different values of the $\beta$ factor, namely $\beta=0.1,0.5,0.9$ and we study how the cross section depends on the energy of the particle and the scattering angle.

The formula used was the following, in natural units:

$$
\frac{d \sigma}{d \Omega}=\frac{8 M^{2} \cdot 4 \pi \alpha}{\beta^{2} E^{2} \sin ^{2} \frac{\theta}{2}}
$$

At first we consider an electron, having the magnetization $M$ equal to the value of the Bohr magneton, in natural units, $M=5.788 \cdot 10^{-5} \mathrm{eV} \cdot T^{-1}$.

The results presented in figs. (1)) show the differential cross section in terms of energy, observing a clear difference between different values of $\beta$, with smaller values having higher probabilities of scattering. We also find in fig. (2)) that the differential cross section is important for small scattering angles as in the case of Coulomb scattering.

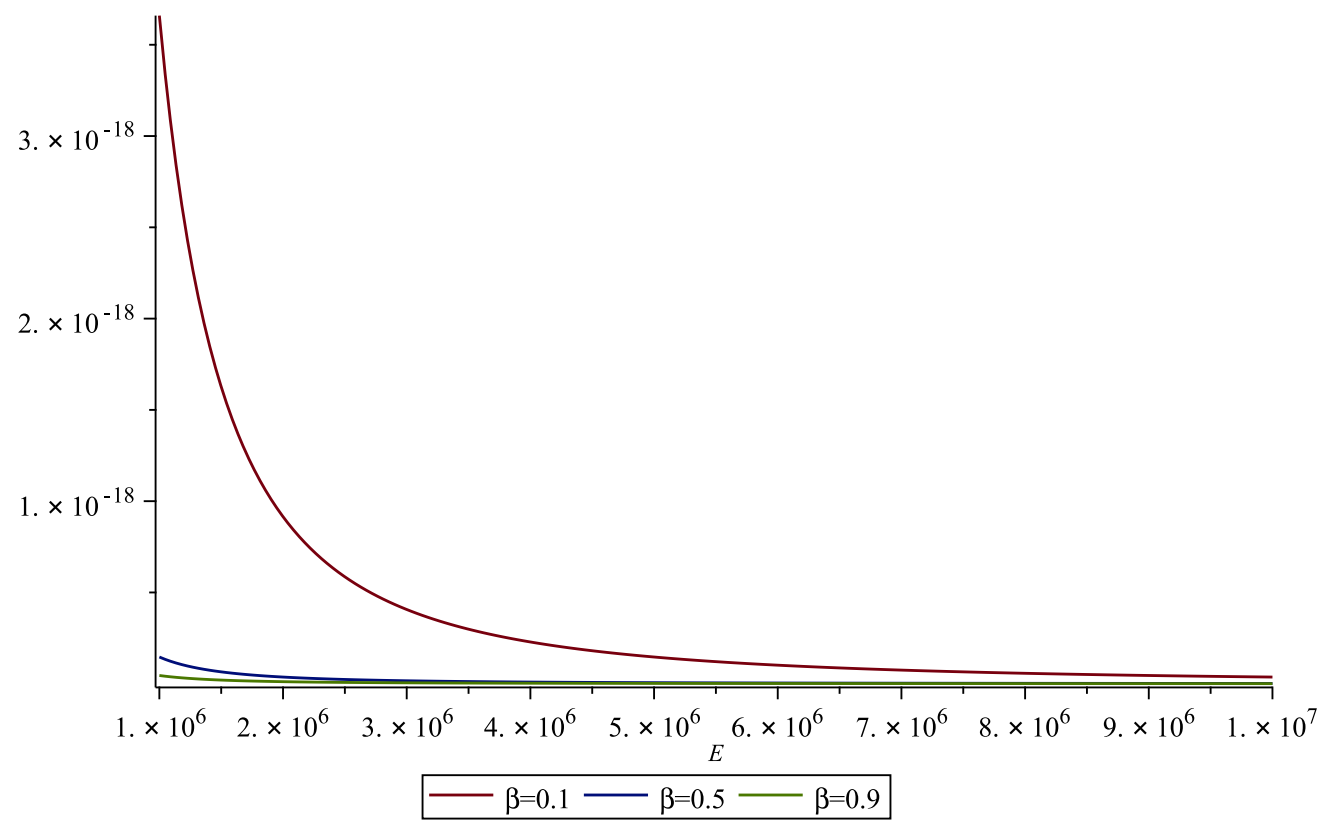

Figure 1. The differential cross section, considering the Bohr magneton and three values of $\beta$ at an angle of $\theta=\frac{\pi}{6}$ 


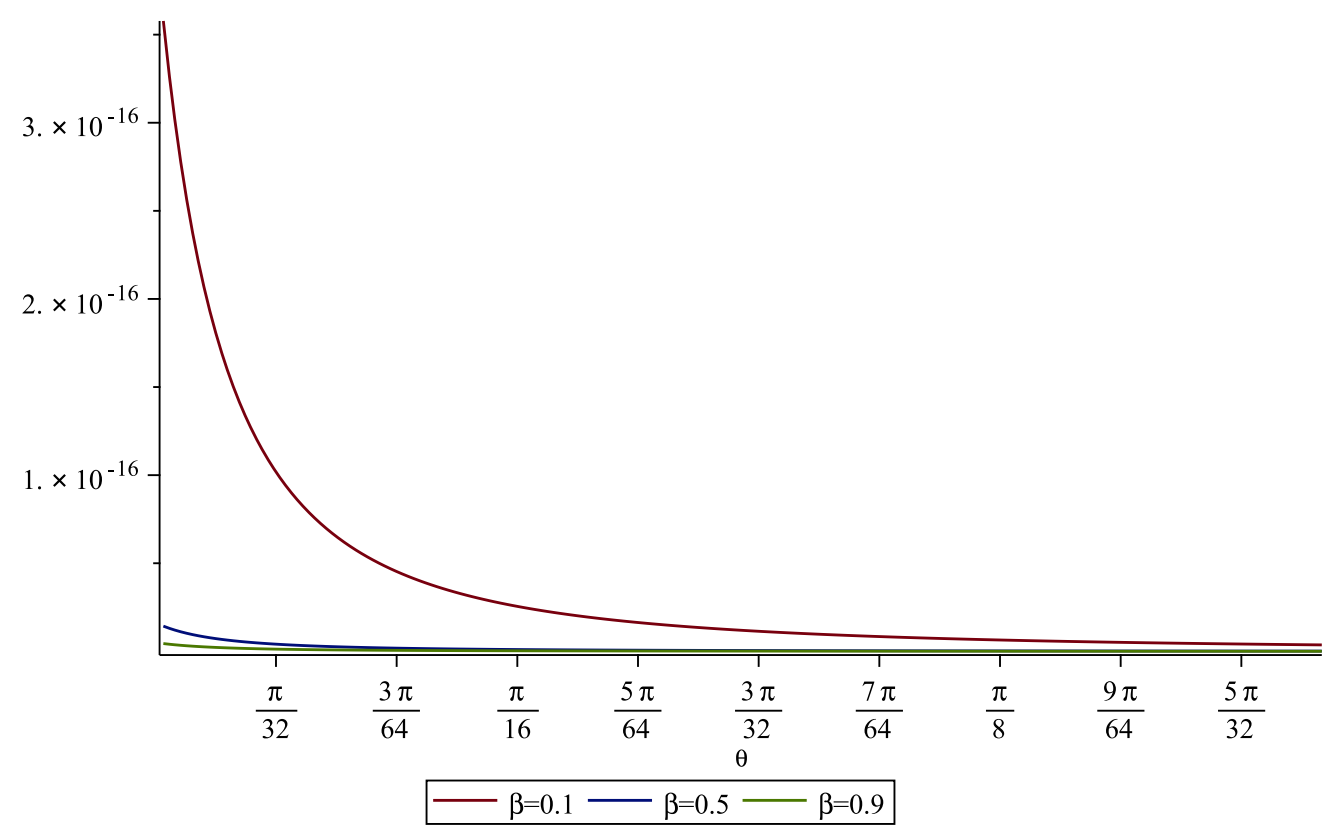

Figure 2. The differential cross section, considering the Bohr magneton and three values of $\beta$ at an energy of $E=1 \mathrm{MeV}$

In order to establish differences between relativistic scattering on a magnetic dipole and Coulomb relativistic scattering, we have chosen the scale of the magnetization to be of the same order, otherwise the comparison would depend solely on the magnetization of the magnetic dipole:

$$
M^{2} \approx \alpha
$$

The formula for the magnetic dipole differential cross section remains the same (25), while the Coulomb scattering cross section is defined as follows:

$$
\frac{d \sigma}{d \Omega}=\frac{\alpha^{2}}{4 E^{2} \beta^{4} \sin ^{4} \frac{\theta}{2}}\left(1-\beta^{2} \sin ^{2} \frac{\theta}{2}\right)
$$

We will focus on the ultrarelativistic regime, where the differences are more pronounced, setting $\beta=0.99$. First we will study the dependence on the scattering angle in fig. (3)), considering a particle with energy of $E=1 \mathrm{MeV}$, and then the dependence on energy in fig. (4)), at a fixed scattering angle of $\theta=\frac{\pi}{6}$. We specify that $\frac{d \sigma}{d \Omega}$ is expressed in $\mathrm{MeV}^{-2}$. 


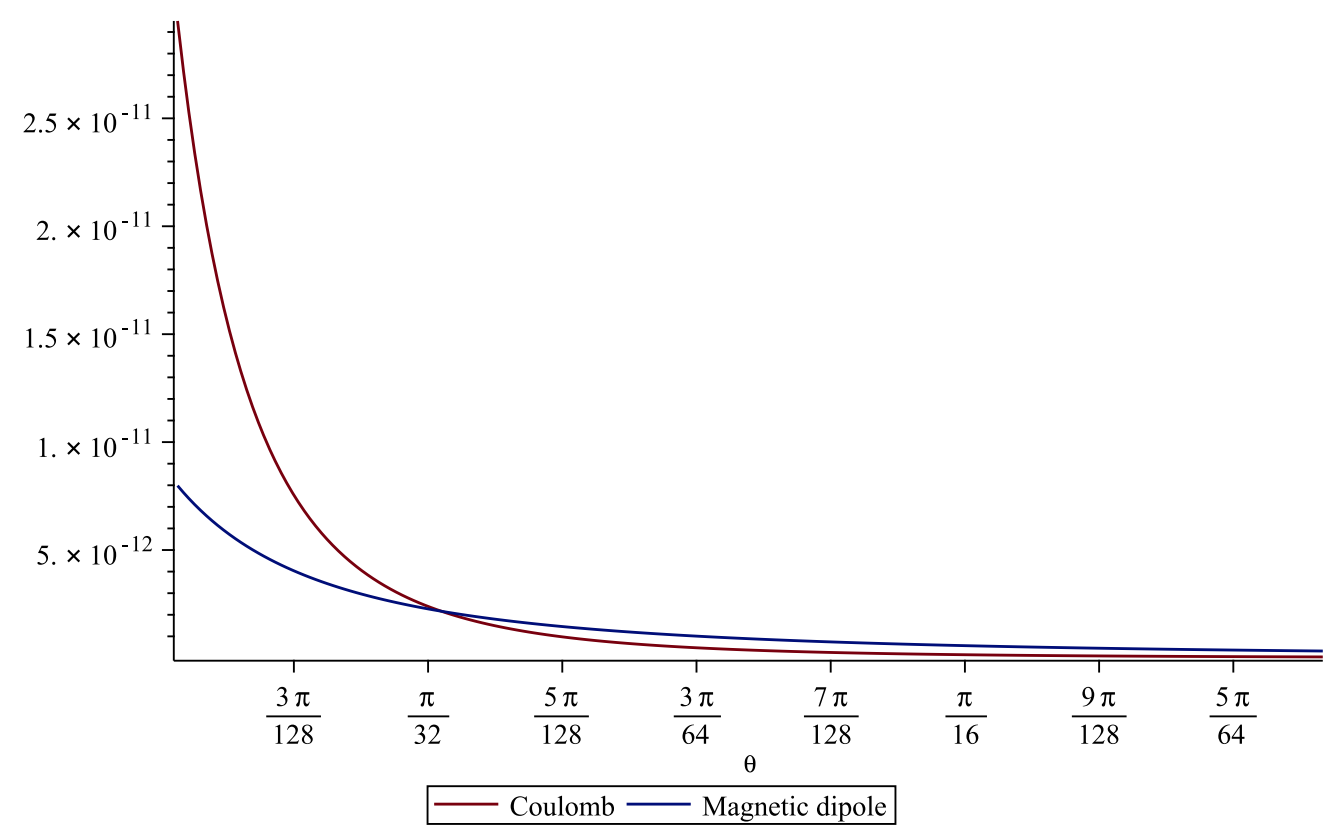

Figure 3. Comparison between Coulomb and magnetic dipole scattering, at values $\beta=0.99$ and $E=1 \mathrm{MeV}$

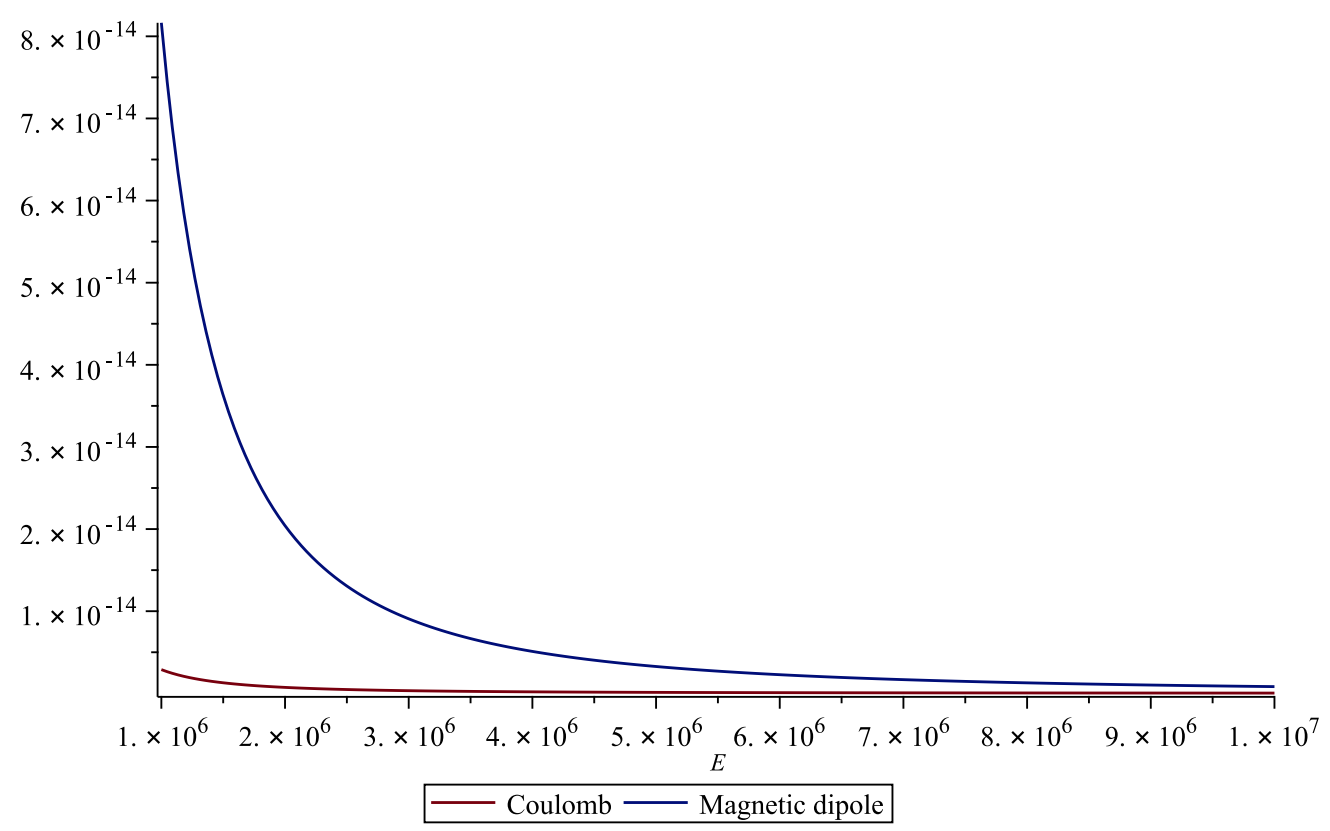

Figure 4. Comparison between Coulomb and magnetic dipole scattering, at values $\beta=0.99$ and $\theta=\frac{\pi}{6}$

In our graphs we consider that the differential cross section is expressed in terms

\section{Conclusions}

In this paper we analyse the problem of fermion scattering on the dipole magnetic field obtaining the differential cross section for the case when the fermions move in the plane perpendicular with the direction of magnetic dipole field. The analysis prove that the differential cross section is significant for small scattering angles even if we consider only the transversal motion for incident fermion and scattered fermion. For further analysis it will be important to 
obtain the differential cross section in the general case by taking into account the non-transversal motion.

\section{References}

[1] A. Zazunov, A. Kundu, A. Hütten, R. Egger, Phys. Rev. B 82, 155431 (2010).

[2] K.M. Whitcomb, D. C. Latimer, American Journal of Physics 85, 932 (2017).

[3] C. Crucean, M. Băloi, Phys. Rev. D 93, 044070 (2016).

[4] S. Weinberg, The Quantum Theory of Fields (Cambridge University Press, Cambridge, 1995); S. Drell and J. D. Bjorken, Relativistic Quantum Fields (Mc Graw-Hill Book Co., New York 1965)

[5] J. D. Jackson ,Classical Electrodynamics, (John Wiley and Sons Ltd. 1962); W. Greiner, Classical Electrodynamics, (Springer 1998). 